\title{
Detection of a common chimeric transcript between human chromosomes 7 and 16
}

\author{
Wenwen Fang ${ }^{1}$, Yong Wei $^{1}$, Yibin Kang ${ }^{1}$ and Laura F Landweber ${ }^{2 *}$
}

\begin{abstract}
Interchromosomal chimeric RNA molecules are often transcription products from genomic rearrangement in cancerous cells. Here we report the computational detection of an interchromosomal RNA fusion between ZC3HAV1L and CHMP1A from RNA-seq data of normal human mammary epithelial cells, and experimental confirmation of the chimeric transcript in multiple human cells and tissues. Our experimental characterization also detected three variants of the ZC3HAVIL-CHMP1A chimeric RNA, suggesting that these genes are involved in complex splicing. The fusion sequence at the novel exon-exon boundary, and the absence of corresponding DNA rearrangement suggest that this chimeric RNA is likely produced by trans-splicing in human cells.

Reviewers: This article was reviewed by Rory Johnson (nominated by Fyodor Kondrashov); Gal Avital and Itai Yanai

Keywords: Chimeric transcripts, RNA fusion, trans-splicing, Genome rearrangement
\end{abstract}

\section{Findings}

High-throughput sequencing techniques have allowed characterization of genome and transcriptome catalogs in an unprecedented detail, revealing complex structures of genome rearrangements [1] and transcript networks of chimeric RNAs in human cells [2]. Because genome instability is a hallmark of cancer [3], most studies of genome rearrangements and RNA fusions focus on cancer cells, and some chimeric RNAs appear to result from DNA rearrangements [4-8]. In fact, some studies use RNA-seq data as a guide to annotate genome rearrangement [9]. Several findings, however, suggest that normal human cells also produce chimeric RNA through transsplicing [10-13]. Notably, Li et al. demonstrated that in normal endometrial cells, trans-splicing produces a chimeric RNA that is identical to a fusion transcript present in endometrial stromal tumor cells [13]. The corresponding chromosomal translocation which may permit production of this chimeric RNA by cis-splicing is present in endometrial tumor cells, but not detectable in normal endometrial cells. This hints at the possibility that RNA fusion may even predispose relevant genomic loci to rearrangement $[13,14]$, via RNA-guided DNA recombination, which our lab previously discovered in the ciliate Oxytricha [15].

\footnotetext{
* Correspondence: Ifl@princeton.edu

${ }^{2}$ Department of Ecology and Evolutionary Biology, Princeton University, Princeton, NJ 08544, USA

Full list of author information is available at the end of the article
}

We therefore asked whether we can identify more occurrences of chimeric transcripts, especially those involving genes on separate chromosomes, in normal human cells. We mined high-throughput RNA-seq data from human mammary epithelial cells (HMEC) available from the ENCODE project [16]. The deFuse program [17] predicted one interchromosomal RNA fusion between the genes encoding ZC3HAV1L (zinc finger $\mathrm{CCCH}$-type, antiviral 1-like) and CHMP1A (charged multivesicular body protein $1 \mathrm{~A}$ ), located on chromosomes 7 and 16, respectively (Figure 1A). ZC3HAV1L contains 5 exons and encodes a 300 residue protein. CHMP1A has two protein-coding transcript isoforms, according to NCBI annotation; transcript variant 1 contains 6 exons encoding a 240 residue protein, and transcript variant 2 contains 7 exons encoding a 196 residue protein, which functions as a tumor suppressor in human kidney and pancreas [18,19]. CHMP1A isoform 1 skips exon 2, but has a larger exon 7. Here we used CHMP1A isoform 1 as the reference for annotation purposes, because the fusion product we detect contains the larger exon 7 (see below).

Because we initially predicted the presence of the ZC3HAV1L-CHMP1A fusion in a breast cell line, we first verified the presence of this chimeric transcript in human mammary cells. Use of a primer pair (Table 1) that amplifies across the predicted ZC3HAV1L-CHMP1A fusion junction (from ZC3HAV1L exon 2 to CHMP1A exon 6) confirms the presence of this fusion at the RNA 


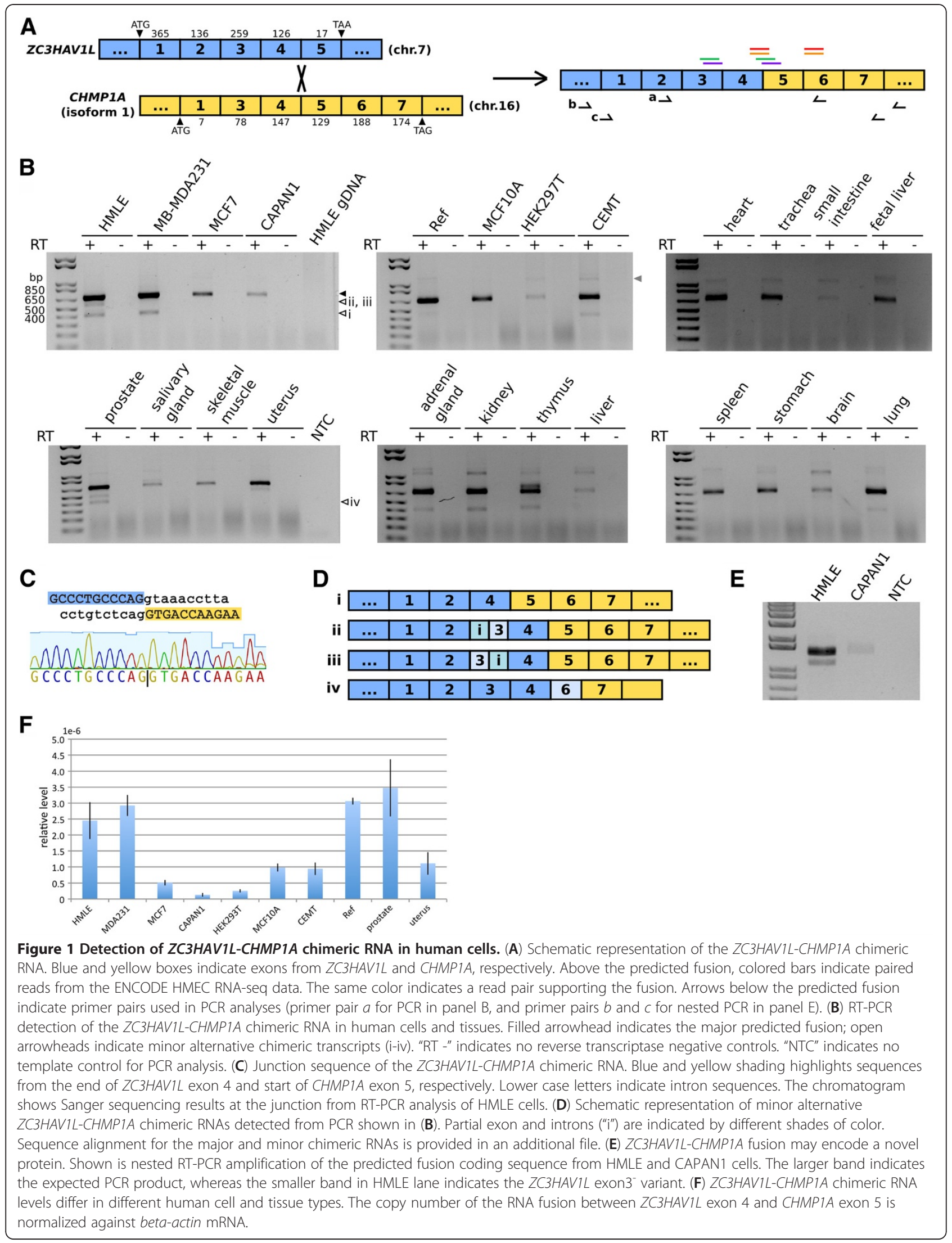


Table 1 Oligonucleotide sequences $\left(5^{\prime}-\mathbf{3}^{\prime}\right)$

\begin{tabular}{ll}
\hline ZC3HAV1L_exon2_F & TGGTCTCAATGAAAACCAGCTTCGG \\
CHMP1A_exon6_R & ATTCTCCTCGGCGATCTGCATGATG \\
ZC3HAV1L_nested_F & AGCGACCATGGCGGAGCCCA \\
CHMP1A_nested_R & CACCGCCCAACCTAAAAGAACAGG \\
ZC3HAV1L_Cds_F & ATGGCGGAGCCCACAGTGTGCTCC \\
CHMP1A_cds_R & CTAAGGCCACGCAGGCCTGGCAG \\
ZC3HAV1L_qPCR_F & AGAAGCTGGTCCTCTGGCTTCTGT \\
CHMP1A_qPCR_R & TCACCTGGGCCATATTCTTGTCA \\
ACTB_qPCR_F & GCACAGAGCCTCGCCTT \\
ACTB_qPCR_R & CCTTGCACATGCCGGAG \\
\hline
\end{tabular}

level in HMLE cells, which are human mammary cells derived from HMEC, but not at the DNA level from matching genomic DNA (Figure 1B). Sequencing of the PCR product verified the fusion junction (Figure 1C). The same PCR analysis suggests that the ZC3HAV1L-CHMP1A fusion is present in MCF10A, an immortalized but otherwise normal human mammary epithelial cell line, as well as two human breast cancer cell lines, MB-MDA231 and MCF7. We also detected the fusion in CAPAN1, a pancreatic cancer cell line, human embryonic kidney (HEK) $293 \mathrm{~T}$ cells, and CEMT, a human T cell line. In addition, we were able to amplify the fusion RNA from commerciallyavailable human universal reference RNA and a panel of human tissue RNAs (Figure 1B). These results suggest that the ZC3HAV1L-CHMP1A chimeric RNA is common across multiple human tissue types, both healthy and diseased.

Curiously, we detected some minor PCR products of different sizes as well. Sequencing revealed that some of them reflect alternative splicing of the ZC3HAV1L-CHMP1A chimeric RNA, adding additional complexity to this fusion transcript. A common splicing variant present in multiple tissue types is the full-length chimeric RNA skipping ZC3HAV1L exon 3 (Figure 1B, D). Two other minor RT-PCR products suggest splicing between partial intron 2 and exon 3, and partial exon 3 and intron 3 of ZC3HAV1L, respectively (Figure 1B, D). Sequence alignments (see Additional file 1) indicate that these alternative splicing events all occur at canonical splicing sites, suggesting that they likely derive from authentic, alternative RNA splicing, rather than an in vitro RT-PCR artifact. A fusion product detected from RT-PCR analysis of human prostate RNA fuses ZC3HAV1L exon 4 to part of CHMP1A exon 6, not at canonical splicing site but between a pair of 5 bp direct repeats at the boundary. Therefore, this may represent either an endogenous activity or just template-switching during the reverse transcription step in our experimental procedure.

The major chimeric, fusion RNA that joins exon 4 of ZC3HAV1L to exon 5 of CHMP1A preserves the open reading frame. We therefore tested whether the entire open reading frame could be detected from mRNA by nested PCR. The use of primers located upstream and downstream of the predicted start and stop codons in the first round of PCR, and then a nested primer pair between the start and stop codons did amplify a product containing the predicted open reading frame, as well as the ZC3HAV1L exon3 version of the transcript, though at much lower levels, consistent with our previous PCR result (Figure 1E). We infer that the major chimeric RNA that joins ZC3HAV1L and CHMP1A may encode a novel fusion protein. Predicted domains are not available for either fusion partner, however, precluding further structural and functional predictions of this putative fusion protein.

From qPCR analysis, we estimated that the ZC3HAV1LCHMP1A chimeric RNA is present at $\sim 0.1$ copies per HMLE cell, suggesting that its expression is limited to either a small population of cells, or a transient time window. We also assayed the relative abundance of this chimeric RNA compared to beta-actin, a constitutively expressed gene, and found that the relative levels of the ZC3HAV1L-CHMP1A chimeric RNA differ in different samples (Figure 1F). This suggests that the production of ZC3HAV1L-CHMP1A RNA might be regulated, or it might be a stochastic event.

In summary, we report the discovery of a chimeric RNA between ZC3HAV1L and CHMP1A in human, located on chromosome 7 and 16, respectively. The fusion occurs at an exon-exon boundary, and was detected both computationally and experimentally from different cells or tissue types. This suggests it is not an artifact from reverse transcription, and is likely an authentic trans-splicing product. We also detected three minor variants which also likely result from trans-splicing, because the fusion occurs at canonical "GT-AG" splicing sites. The fusion products are present at very low levels, and thus may reflect promiscuous splicing involving ZC3HAV1L and CHMP1A.

Could such low abundance chimeric RNAs have any function? While the major chimeric RNA that we detected preserves open reading frame and could potentially produce a novel fusion protein or proteins, we propose that such examples of chimeric RNA may also occasionally impact somatic genome rearrangements, facilitating rogue recombination events between the two respective chromosomes [14,15]. The ability of RNA to influence genome remodeling has gained considerable support and interest over the past few years [20], but RNA-guided DNA rearrangement in humans still needs further investigation, emphasizing the importance of detecting more chimeric RNAs and their possible DNA rearrangements in normal or diseased tissue. 


\section{Methods}

\section{Computational detection of fusion RNAs}

HMEC polyA-RNA-seq data from ENCODE project (http://hgdownload.cse.ucsc.edu/goldenPath/hg19/encodeDCC/wgEncodeCshlLongRnaSeq/) were downloaded from UCSC genome browser website. DeFuse 0.4.3 [17] was used to detect fusion transcripts, requiring the presence of two pairs of spanning reads and one split read at the junction. We divided the RNA-seq data into subsets of 10 and 20 million paired-end reads to accommodate computation memory, and the ZC3HAV1L-CHMP1A fusion was predicted in two independent subsets of data.

\section{RNA and genomic DNA extraction}

Total RNA from HMLE, MB-MDA231, MCF7, CAPAN1, MCF10A, HEK293T, and CEMT cells was extracted using RNeasy (Qiagen), and DNase treated with TURBO DNAfree kit (Ambion) following the manufacturer's instructions. Human reference RNA was purchased from Stratagene, and the human tissue RNA panel was purchased from Clontech. Genomic DNA was extracted using NucleoSpin Tissue (Macherey-Nagel).

\section{CDNA synthesis}

$3.5 \mu \mathrm{g}$ of RNA was reverse transcribed with SuperScriptIII reverse transcriptase (Invitrogen), in a $20 \mu \mathrm{l}$ reaction following the oligo(dT) priming protocol.

\section{PCR analysis}

FastStart High Fidelity PCR system (Roche) was used to amplify fusion product from $1 \mu \mathrm{l}$ of cDNA (equivalent of 175 ng RNA), or 200 ng genomic DNA. To detect the predicted fusion from $Z C 3 H A V 1 L$ exon 2 to $C H M P 1 A$ exon 6 , the following program was used: $95^{\circ} \mathrm{C} 2 \mathrm{~min}$ initial denaturing; $95^{\circ} \mathrm{C} 30 \mathrm{~s}, 58^{\circ} \mathrm{C} 30 \mathrm{~s}, 72^{\circ} \mathrm{C} 45 \mathrm{~s}$ for 36 cycles; $72^{\circ} \mathrm{C} 7 \mathrm{~min}$. To recover the entire coding region by nested PCR, the following program was used. First round: $95^{\circ} \mathrm{C} 2$ min initial denaturing; $95^{\circ} \mathrm{C} 30 \mathrm{~s}$, $60^{\circ} \mathrm{C} 30 \mathrm{~s}, 72^{\circ} \mathrm{C} 90 \mathrm{~s}$ for 20 cycles; $72^{\circ} \mathrm{C} 7 \mathrm{~min}$. The PCR reaction was diluted 100 fold, and used in the second round of PCR: $95^{\circ} \mathrm{C} 2 \mathrm{~min}$ initial denaturing; $64^{\circ} \mathrm{C} 30 \mathrm{~s}$, $72^{\circ} \mathrm{C} 80 \mathrm{~s}$ for 30 cycles; $72^{\circ} \mathrm{C} 7 \mathrm{~min}$.

\section{Sanger sequencing of $P C R$ products}

PCR products were either sent for direct Sanger sequencing (Genewiz) following Genewiz DNA sequencing instructions, or TOPO-cloned (Invitrogen) for colony PCR and sequencing (Genewiz).

\section{qPCR analysis}

The 7900HT Fast Real-Time PCR System and SYBR green master mix (Applied Biosystems) were used for qPCR analysis with the default cycling program. Standard curves for each primer pair were generated with five ten-fold serial dilutions of appropriate control plasmids in yeast RNA, allowing absolute quantification of DNA levels. Primer specificity was confirmed by a meltcurve analysis. Each primer pair detected the full range of standards with a correlation of $\mathrm{R}^{2}>0.99$.

\section{Reviewers' comments}

Review by Rory Johnson (Centre de Regulacio Genomica, nominated by Fyodor Kondrashov)

The authors report a new transcript resulting from a fusion of two genes on distinct chromosomes. The range of computational and experimental evidence support the notion that this transcript results from a trans-splicing event rather than from chromosomal translocation or technical artifacts. The manuscript is clear and well presented. I do not have any technical or theoretical arguments with this work. I only have one suggestion: If the fusion transcript has any biological function/phenotype, one might expect that it carries functional domains from its two constituent genes. Therefore, one might expect the fusion junction to occur at linker regions of functional domains, and not interrupting the amino acid sequence in the middle of such a domain. Therefore, I would suggest that the authors comment on where the fusion occurs for both the protein coding sequences with respect to their predicted functional domains, AND include a diagram of the predicted domain structure of the protein encoded by the fusion transcript. E.g. does the fusion carry the zinc finger domain of ZC3HAV1L? What is known about the functions of the two parent protein coding genes?

Author's response: We did not find any domain annotation for ZC3HAV1L or CHMP1A in Pfam. Despite its name, ZC3HAV1L is actually not a zinc-finger protein. Therefore we did not pursue further the functional predictions of the putative fusion protein. In the revised manuscript we included a comment on this subject.

\section{Review by Gal Avital and Itai Yanai, Department of Biology, Technion - Israel Institute of Technology}

In this report, Fang et al. report a transcript that appears to be trans-spliced between RNAs originating from two different human chromosomes. Four paired-end reads alone appear to support the chimeral transcript in the examined ENCODE RNA-Seq data for one cell type (HMEC). The PCR analysis implicates other cell types, however, do any of these have RNA-Seq datasets in ENCODE to further support this? The article mentions two possible avenues for function following from such a chimera: 1 . a protein, and 2 . an RNA that might serve to proximally position chromosomes. While the transcript has some of the hallmarks of real transcripts such as the existence of splice variants and an exon-exon boundary, the low expression level does little to support a functional 
protein. Does the fusion disrupt a functional domain in a putative protein? This can be checked by matching with PFAM. For the second option, structural analysis can be used to query for a stable fold. Overall, while the authors have clearly gone to lengths to detect the chimera in various tissues, a possible function would require additional evidence.

Author's response: We further analyzed 40 and 20 million paired-end RNA-seq reads for normal lung and placenta from ENCODE data, respectively, but did not find evidence of the ZC3HAV1L-CHMP1A RNA fusion. Our quantitative RT-PCR analysis suggests that levels of the RNA fusion in HMEC are higher than in most other tissues except for prostate, but we have not found appropriate RNA-seq data from normal prostate tissue to use in computational analysis.

We agree that the low level of the chimeric transcript suggests that it more likely has a non-coding function, that could potentially influence genomic rearrangement. We still leave open the possibility of protein-coding function, since the fusion RNA indeed preserves an open reading frame. As we indicated in response to the first review, there is no domain annotation or relevant structural study for these two partner proteins, and therefore we do not further characterize the putative fusion protein at this time.

\section{Additional file}

Additional file 1: Alignment of the major and minor chimeric transcripts detected in RT-PCR analyses. The predicted chimeric RNA sequence is indicated in the top row, with annotations of exons below it. Fusion coding sequences (CDS) and the exon $3^{-}$variant (variant i) are from nested PCR analysis of HMLE CDNA. Variants ii and iii are from PCR analysis of HMLE CDNA, across ZC3HAV1L exon 2 and CHMP1A exon 6, with partial introns indicated below. Variant iv is from PCR analysis of human prostate CDNA, across ZC3HAVIL exon 2 and CHMP1A exon 6. The annotations are based on [Genbank: NC_000007 REGION: complement (138710452..138720775) GPC_000000031, NM_080660.3, NM_001083314.2].

\section{Abbreviations}

ZC3HAV1L: Zinc finger CCCH-type antiviral 1-like; CHMP1A: Charged multivesicular body protein 1A; HMEC: Human mammary epithelial cell; HEK293T: Human embryonic kidney 293 T; qPCR: Quantitative PCR; gDNA: Genomic DNA.

\section{Competing interests}

The authors declare that they have no competing interests.

\section{Authors' contributions}

WF performed computational analysis of RNA-seq data, and experimental characterization of the chimeric RNAs. All authors participated in project design and manuscript writing. All authors read and approved the final manuscript.

\section{Acknowledgements}

We thank data producers from the ENCODE Consortium for HMEC RNA-seq data; R. Weinberg lab (Whitehead Institute) for sharing HMLE cells, and T. Li for sharing HEK293T and CEMT cells; and A. McPherson, E. Dolzhenko, A. D. Goldman and X. Chen for computational advice. This study was supported by NIH grant GM59708 and NSF grants 0923810 and 0900544 (to L.F.L.); Brewster Foundation, the Champalimaud Foundation, NIH grants R01CA134519 and R01CA141062 (to Y.K.); and a DOD BCRP pre-doctoral fellowship

W81XWH-10-1-0122 (to W.F.)

\section{Author details}

'Department of Molecular Biology, Princeton University, Princeton, NJ 08544, USA. ${ }^{2}$ Department of Ecology and Evolutionary Biology, Princeton University, Princeton, NJ 08544, USA.

Received: 5 September 2012 Accepted: 11 December 2012

Published: 29 December 2012

\section{References}

1. Maher CA, Wilson RK: Chromothripsis and human disease: piecing together the shattering process. Cell 2012, 148:29-32.

2. Djebali S, Lagarde J, Kapranov P, Lacroix V, Borel C, Mudge JM, Howald C, Foissac S, Ucla C, Chrast J, et al: Evidence for transcript networks composed of chimeric RNAs in human cells. PLoS One 2012, 7:e28213.

3. Hanahan D, Weinberg RA: Hallmarks of cancer: the next generation. Cell 2011, 144:646-674.

4. Pflueger D, Terry S, Sboner A, Habegger L, Esgueva R, Lin PC, Svensson MA, Kitabayashi N, Moss BJ, MacDonald TY, et al: Discovery of non-ETS gene fusions in human prostate cancer using next-generation RNA sequencing. Genome Res 2011, 21:56-67.

5. Maher CA, Kumar-Sinha C, Cao X, Kalyana-Sundaram S, Han B, Jing X, Sam L, Barrette T, Palanisamy N, Chinnaiyan AM: Transcriptome sequencing to detect gene fusions in cancer. Nature 2009, 458:97-101.

6. Maher CA, Palanisamy N, Brenner JC, Cao X, Kalyana-Sundaram S, Luo S, Khrebtukova I, Barrette TR, Grasso C, Yu J, et al: Chimeric transcript discovery by paired-end transcriptome sequencing. Proc Natl Acad Sci U S A 2009, 106:12353-12358.

7. Edgren H, Murumagi A, Kangaspeska S, Nicorici D, Hongisto V, Kleivi K, Rye IH, Nyberg S, Wolf M, Borresen-Dale AL, Kallioniemi O: Identification of fusion genes in breast cancer by paired-end RNA-sequencing. Genome Biol 2011, 12:R6.

8. Ruan Y, Ooi HS, Choo SW, Chiu KP, Zhao XD, Srinivasan KG, Yao F, Choo CY, Liu J, Ariyaratne $P$, et al: Fusion transcripts and transcribed retrotransposed loci discovered through comprehensive transcriptome analysis using Paired-End diTags (PETs). Genome Res 2007, 17:828-838.

9. Zhao Q, Caballero OL, Levy S, Stevenson BJ, Iseli C, De Souza SJ, Galante PA, Busam D, Leversha MA, Chadalavada K, et al: Transcriptome-guided characterization of genomic rearrangements in a breast cancer cell line. Proc Natl Acad Sci U S A 2009, 106:1886-1891.

10. Li BL, Li XL, Duan ZJ, Lee O, Lin S, Ma ZM, Chang CC, Yang XY, Park JP, Mohandas TK, et al: Human acyl-CoA:cholesterol acyltransferase-1 (ACAT-1) gene organization and evidence that the 4.3-kilobase ACAT-1 mRNA is produced from two different chromosomes. J Biol Chem 1999, 274:11060-11071.

11. Hahn Y, Bera TK, Gehlhaus K, Kirsch IR, Pastan IH, Lee B: Finding fusion genes resulting from chromosome rearrangement by analyzing the expressed sequence databases. Proc Natl Acad Sci U S A 2004, 101:13257-13261.

12. Herai RH, Yamagishi ME: Detection of human interchromosomal transsplicing in sequence databanks. Brief Bioinform 2010, 11:198-209.

13. Li H, Wang J, Mor G, Sklar J: A neoplastic gene fusion mimics transsplicing of RNAs in normal human cells. Science 2008, 321:1357-1361.

14. Rowley JD, Blumenthal T: Medicine. The cart before the horse. Science 2008, 321:1302-1304.

15. Nowacki M, Vijayan V, Zhou Y, Schotanus K, Doak TG, Landweber LF: RNA-mediated epigenetic programming of a genome-rearrangement pathway. Nature 2008, 451:153-158.

16. Birney E, Stamatoyannopoulos JA, Dutta A, Guigo R, Gingeras TR, Margulies EH, Weng Z, Snyder M, Dermitzakis ET, Thurman RE, et al: Identification and analysis of functional elements in $1 \%$ of the human genome by the ENCODE pilot project. Nature 2007, 447:799-816.

17. McPherson A, Hormozdiari F, Zayed A, Giuliany R, Ha G, Sun MG, Griffith $M_{\text {, }}$ Heravi Moussavi A, Senz J, Melnyk N, et al: deFuse: an algorithm for gene fusion discovery in tumor RNA-Seq data. PLoS Comput Biol 2011, 7:e1001138. 
18. You Z, Xin Y, Liu Y, Sun J, Zhou G, Gao H, Xu P, Chen Y, Chen G, Zhang L, et al: Chmp1A acts as a tumor suppressor gene that inhibits proliferation of renal cell carcinoma. Cancer Lett 2012, 319:190-196.

19. Li J, Belogortseva N, Porter D, Park M: Chmp1A functions as a novel tumor suppressor gene in human embryonic kidney and ductal pancreatic tumor cells. Cell Cycle 2008, 7:2886-2893.

20. Nowacki M, Shetty K, Landweber LF: RNA-mediated epigenetic programming of genome rearrangements. Annual Rev Genomics Human Genet 2011, 12:367-389.

doi:10.1186/1745-6150-7-49

Cite this article as: Fang et al:: Detection of a common chimeric

transcript between human chromosomes 7 and 16. Biology Direct 2012 7:49.

\section{Submit your next manuscript to BioMed Central and take full advantage of:}

- Convenient online submission

- Thorough peer review

- No space constraints or color figure charges

- Immediate publication on acceptance

- Inclusion in PubMed, CAS, Scopus and Google Scholar

- Research which is freely available for redistribution 